\title{
Premature Restriction of Foramen Ovale in an Anemic Fetus From a Rhesus-negative Mother: A Case Report
}

\author{
Homeira Vafaei ${ }^{1}$, Nasrin Asadi', Ali Mohammad Shakibafard ${ }^{2}$, Maryam Kasraeian', Neda Rahimirad ${ }^{1}$, \\ Shaghayegh Moradi Alamdarloo ${ }^{3}$, Shohreh Roozmeh ${ }^{1 * \mathbb{1}}$, Kamran Hessami ${ }^{4}$
}

\begin{abstract}
The fetal middle cerebral artery-peak systolic velocity (MCA-PSV) is a noninvasive tool for detection of intrauterine fetal anemia. Premature restriction of foramen ovale (FO) may prevent proper blood supply to the fetal brain. We presented a 29-year-old Rhesus-negative mother who had been referred to our center due to positive indirect Coombs test. A single live intrauterine fetus at 30 weeks of gestation was observed with normal MCA-PSV- multiples of the median (MoM). The mother was under weekly follow up due to an isoimmunized pregnancy. In the third week of followup, the MCA-PSV- MoM was within the normal range though fetal echocardiography showed that the findings were in favor of premature restriction of FO; so premature delivery was decided at the same day. A boy was delivered with significant paleness, hemoglobin level of $3 \mathrm{~g} / \mathrm{dL}$, positive direct Coombs test, and reticulocyte count of $20 \%$. These findings resulted in a significant intrauterine hemolysis process due to Rh alloimmunization not detected by Doppler examination of MCA- PSV during pregnancy. The aim of this study was to show that MCA-PSV- MoM may not be a diagnostic method for detection of possible anemia in the fetus with congenital heart disease.

Keywords: Fetus, Anemia, Middle cerebral artery, Congenital heart disease, Rhesus factor, Erythroblastosis
\end{abstract}

\section{Introduction}

Rhesus (Rh) negative women who are exposed to Rhpositive red blood cells (RBCs) (e.g., those who deliver a D-positive baby) are at the risk of developing anti-D antibodies against the $\mathrm{Rh}$ antigens present on the surface of RBCs. Memory response of maternal immune system in subsequent re-exposures makes Ig $\mathrm{G}$ antibody which is transferred across the placenta into the fetal circulation. Rh-positive fetuses from these mothers are at the risk of serious morbidity and mortality, and a group of disorders such as severe hemolytic anemia, hydrops fetalis, and intrauterine fetal demise if not treated. The fetal middle cerebral artery-peak systolic velocity (MCA-PSV) is a noninvasive tool for the diagnosis of fetal anemia. The sensitivity of MCA-PSV> $1.5 \mathrm{MoM}$ (multiples of the median) is $100 \%$ for the prediction of moderate or severe anemia in the fetuses never transfused with a false positive rate of $12 \%$ (1). During intrauterine life, foramen ovale (FO) is the only pathway for the oxygenated maternal blood to enter from the right atrium of the heart to the left one and then being pumped into the upper part of fetus's body (2). Premature closure or restriction of the FO is a rare and serious clinical condition that prevents this normal fetal blood circulation. It can be associated with fetal arrhythmia, right -side heart failure, pericardial effusion, tricuspid regurgitation (TR), non-immune hydrops fetalis, and intrauterine fetal death with unknown causes during the pregnancy $(3,4)$. Premature restriction of FO is diagnosed based on the following criteria (5):

- $\quad$ An FO diameter $<3 \mathrm{~mm}$ with a Doppler velocity measured gradient $>5 \mathrm{~mm} \mathrm{Hg}$ or

- $\quad$ FO diameter $<2 \mathrm{~mm}$ with Doppler velocity $>120$ $\mathrm{cm} / \mathrm{s}$

In this study, a case of premature restriction of $\mathrm{FO}$ was described in a fetus from the $\mathrm{RH}$ alloimmunizated mother who had severe intrauterine hemolytic anemia not detected antepartum by routine Doppler ultrasound examination of MCA-PSV-MoM..

\section{Case Report}

A 29-year-old woman with G3D1L1 (two previous cesarean sections) and $\mathrm{Rh}$ alloimmunization was referred to the Hafez High-Risk Perinatology Center affiliated to Shiraz University of Medical Sciences, Shiraz, Fars province, Iran due to indirect Coombs titer of 1.458 on July 10, 2019. After providing an informed consent from mother we presented here. A single live intrauterine fetus at 30 weeks of gestation was observed with MCA-PSV of 42 and a MoM of 1.20. Two-dimensional ultrasound examination showed a mild pericardial effusion, increased heart size with a cardiothoracic area ratio of 0.45 , and significant TR; however, there was no definite sign of hydrops fetalis. The mother and her fetus were under outpatient follow-up with weekly fetal Doppler sonography and fetal echocardiography as an isoimmunized pregnancy. In the third week of follow -up, the findings of fetal 
echocardiography examination compared to previous findings showed:

1. Increased size of the right atrium with left bulging of the foramen ovale without an evident flap,

2. Restricted flow from right atrium to the left,

3. Significant TR,

4. A significant narrowing of the aortic arch due to low blood flow velocity,

5. Dilated inferior vena cava (IVC) and superior vena cava (SVC) due to the increased preload.

These findings were in favor of premature restriction of FO (see the related video). At that time, OB Doppler sonography revealed a fetus at 32 weeks of gestation, which was correlated with first trimester scan, with the MCA-PSV of 57 and MoM of 1.28, which were within the normal range again. Above echocardiography and abnormal cardiotocography findings led us to the premature birth at the same day. A boy was delivered with significant paleness and bradycardia with a 5-minute Apgar score of 7 and birth weight of $2080 \mathrm{~g}$. The results of newborn screening were as follows: $\mathrm{Rh}$ positive, Hemoglobin level of $3 \mathrm{~g} / \mathrm{dL}$, Hematocrit level of 11.2, positive direct Coombs test, total bilirubin concentration of $12 \mathrm{mg} / \mathrm{dL}$, and reticulocyte count of $20 \%$. These results were obtained from a significant intrauterine hemolysis process, as well as fetal anemia due to $\mathrm{Rh}$ alloimmunization not detected by Doppler sonography examination of MCA-PSV during the pregnancy. The newborn received blood transfusion for correction of anemia. His condition progressed to severe immune -mediated hemolysis and hyperbilirubinemia during the days after delivery. He was treated with partial blood exchange for six times and administration of intravenous immunoglobulin. Finally, the happy baby was discharged from the neonatal intensive care unit after 15 days.

\section{Discussion}

In this case report, a case of premature restriction of FO was described in a fetus from an $\mathrm{Rh}$ alloimmunization with severe intrauterine hemolytic anemia not detected in antenatal care by Doppler ultrasound examination of MCA-PSV. It seems that the premature restriction of the FO in this fetus prevented proper blood flow from the left atrium to the left ventricle and then to the fetus's brain. In this condition, the brain was perfused using a retrograde blood supply by ascending the aorta. As a result, MCA blood flow velocity decreased and MCA-PSV-MoM was measured to be in the normal range. Currently, serial fetal MCA-PSV is considered as a noninvasive tool for the diagnosis of fetal anemia in the fetuses never transfused with a $100 \%$ sensitivity and a false positive rate of $12 \%$. There is limited evidence in the literature about cerebrovascular blood flow response and MCA-PSV measurement associated with congenital heart failure. Jansen et al showed that fetuses with congenital heart defect have a considerable decreased cerebrovascular blood flow, which is due to the intracardiac mixing of oxygenated and deoxygenated blood, rendered as a marker of cerebral hypoxemia (6). Jouannic et al prospectively described 23 fetuses with the prenatal diagnosis of transposition of the great arteries whose median middle cerebral artery pulsatility index was significantly lower than that of the control group $(P<0.001)(7)$.

\section{Conclusion}

Toconclude, itseems that Doppler sonographyexamination of MCA-PSV was not an appropriate diagnostic method for detecting fetal anemia in our presented case. Thus, it is believed that serial cordocentesis may be a better approach for the definitive diagnosis of fetal anemia in the fetuses with congenital heart disease, having restricted left heart blood flow; although further studies are needed.

\section{Authors' Contribution}

$\mathrm{HV}, \mathrm{NA}, \mathrm{SR}, \mathrm{AMS}$ participated in the conceptualization, design, and critical revision of the final manuscript. $\mathrm{HV}, \mathrm{NA}, \mathrm{MK}, \mathrm{NR}, \mathrm{SMA}$ and $\mathrm{KH}$ aided in design, preparation of manuscript and critical revision of the final manuscript. All authors read and approved the final manuscript.

\section{Conflict of Interests}

Authors declare that they have no conflict of interests.

\section{Ethical Issues}

Informed consent was obtained from the patient for publication of the report.

\section{Financial Support}

The study was funded by Shiraz University of Medical Sciences.

\section{References}

1. Pretlove SJ, Fox CE, Khan KS, Kilby MD. Noninvasive methods of detecting fetal anaemia: a systematic review and meta-analysis. Bjog. 2009;116(12):1558-1567. doi:10.1111/j.1471-0528.2009.02255.x

2. Yagel S, Silverman NH, Gembruch U. Fetal Cardiology: Embryology, Genetics, Physiology, Echocardiographic Evaluation, Diagnosis and Perinatal Management of Cardiac Diseases. 3rd ed. Boca Raton: CRC Press; 2018:730.

3. Hernández Herrera RJ, Ochoa Torres M, Ramos González R, Ramírez Sánchez LF. Prenatal diagnosis of foramen ovale premature closure and hydrops fetalis. Diagnóstico Prenatal. 2013;24(4):158-160. doi:10.1016/j.diapre.2013.03.004

4. $\mathrm{Li}$ YD, Li ZA, He YH. Premature closure or restriction of the foramen ovale: prenatal diagnosis by directional enhanced flow imaging. J Ultrasound Med. 2013;32(7):1291-1294. doi:10.7863/ultra.32.7.1291

5. Gupta U, Abdulla RI, Bokowski J. Benign outcome of pulmonary hypertension in neonates with a restrictive patent foramen ovale versus result for neonates with an unrestrictive patent foramen ovale. Pediatr Cardiol. 2011;32(7):972-976. doi:10.1007/s00246-011-0032-7

6. Jansen FA, Everwijn SM, Scheepjens R, et al. Fetal brain imaging in isolated congenital heart defects - a systematic review and metaanalysis. Prenat Diagn. 2016;36(7):601-13. doi: 10.1002/pd.4842.

7. Jouannic JM, Benachi A, Bonnet D, et al. Middle cerebral artery Doppler in fetuses with transposition of the great arteries. Ultrasound Obstet Gynecol. 2002;20(2):122-124. doi:10.1046/j.14690705.2002.00756.x 